\title{
Allergy Consultation via Smart Glasses: Cases Reports of Real-Time Clinical Decision Support for Suspected Oral Metal Allergy
}

\author{
Carsten R Hamann ${ }^{1 *}$, Dathan Hamann², Kylin Sager ${ }^{1}$, Christian Dameff ${ }^{3}$, Jeffrey Tully ${ }^{4,5}$ and Beth Hamann $^{6}$ \\ ${ }^{1}$ Loma Linda University School of Medicine, Loma Linda, CA 92350, United States of America \\ ${ }^{2}$ The Ohio State University, Department of Internal Medicine, Division of Dermatology, Columbus, OH 43221, USA \\ ${ }^{3}$ Maricopa Medical Center, Department of Emergency Medicine, 2601 E Roosevelt St. Phoenix, AZ 85008, USA \\ ${ }^{4}$ Phoenix Children's Hospital, 1919 E. Thomas Rd. Phoenix, AZ 85006, USA \\ ${ }^{5}$ Maricopa Medical Center, Department of Pediatrics, 2601 E Roosevelt St. Phoenix, AZ 85008, USA \\ ${ }^{6}$ Agave Dental, 4235 N 32nd St. Phoenix, Arizona 85018, USA
}

\begin{abstract}
Objectives: We explore and describe clinical experiences of the early adoption of smart glasses, for dermatology/ allergy decision support in two cases of suspected oral metal allergy.

Materials and methods: Smart glasses were used to facilitate physician-dentist communication during dentalexamination for two patients with concerns for metal allergy and bio-incompatibility.

Results: Clinical use of smart glasses has reduced the burden of multiple visits and facilitated coordinated care for complex patients with suspected oral metal allergy.

Conclusions: Smart glasses technology may be used for hands-free videoconferencing during exams and procedures to coordinate care and consult experts in cutaneous allergy, increasing quality of care in complex cases and decreasing patient consults and visits.

Clinical relevance: Smart glasses and other telecommunication devices will play larger roles in healthcare in the future, especially where their use will decrease visits and potentially decrease cost of care.
\end{abstract}

Keywords: Metal contact allergy; Dentistry-related allergy; Smart glasses; Oral allergy; Implant failure; Dentistry-associated contact allergy; Triage; Tele-dentistry

\section{Introduction}

Telecommunications technologies have been utilized to enhance patient care in healthcare for decades.

We authors enthusiastically relay to the readers of recent fruitful applications of telecommunication technologies in dermatology and allergy settings including decision support and triage for referral to dermatologists for suspected cutaneous neoplasms [1], and general tele-consultation [2]. Recent articles have also highlighted developments in tele-dentistry, for example using technologies to increase dental accessibility in remote areas [3], oral medicine [35], and prosthodontics [3]. In recent years, both dental and medical professionals have used email to aid in diagnosis by consulting with other clinicians or specialists [6,7]. Studies have highlighted the use of intraoral cameras for store-and-forward tele-dentistry, with evidence that this application may provide an acceptable index of correct diagnosis for dentists working with mid-level providers in remote locations [4]. Interestingly, it has also been shown that cell-phone cameras perform well in some tele-dentistry and tele-dermatology applications [8,9]. In addition to store-and-forward applications, video conferencing has also been used in many fields of dentistry including pre-implant assessments, oral surgery, as well as dental education $[5,10,11]$. The value and utility of implementing this technology in the clinical dermatology and allergy settings is still being investigated.

Following in the footsteps of other innovative applications of new telecommunications devices in healthcare, it is our goal to explore early adoption of smart glasses technology for clinical decision support in a dental setting for triaging patients with suspected oral metal allergy. We seek to demonstrate that dentists may be able to communicate with remote colleagues such as allergists or dermatologists effectively in a quick and easy manner to ensure timely and quality decision-making for patients with suspected oral metal allergy.

\section{Case 1}

Smart glasses technology (Google Glass, Google Inc.; Mountain View, CA, USA) has recently been utilized effectively by dentists at Agave Dental in Phoenix, Arizona to consult with medical colleagues on various difficult cases and discuss the suitability of patients for referral. A 53-year-old Caucasian female presented for implant placement. She is a non-smoker, with a history of jewelry avoidance, a common surrogate marker for metal hypersensitivity and has no history of diabetes mellitus, other systemic disease, or immunocompromised state. She presented for placement two grade 5 titanium alloy implants (titanium-aluminum-vanadium) in an edentulous area of the lower left mandible in the area of missing $1^{\text {st }}$ and $2^{\text {nd }}$ permanent molars. Cone-beam computerized tomography sizing was utilized and placement was well-tolerated. Two weekspost placement, the patient returned with significant pain and swelling and complained of limited range of motion of the mandible. Peri-implantitis was noted on exam

*Corresponding author: Carsten R Hamann, Loma Linda University School of Medicine, Loma Linda, CA 92350, United States, Tel: 6023272977; E-mail: hamann511@gmail.com

Received May 19, 2013; Accepted July 23, 2014; Published July 30, 2014

Citation: Hamann CR, Hamann D, Sager K, Dameff C, Tully J, et al. (2014) Allergy Consultation via Smart Glasses: Cases Reports of Real-Time Clinical Decision Support for Suspected Oral Metal Allergy. J Allergy Ther 5: 186. doi:10.4172/21556121.1000186

Copyright: @ 2014 Hamann CR, et al. This is an open-access article distributed under the terms of the Creative Commons Attribution License, which permits unrestricted use, distribution, and reproduction in any medium, provided the original author and source are credited. 


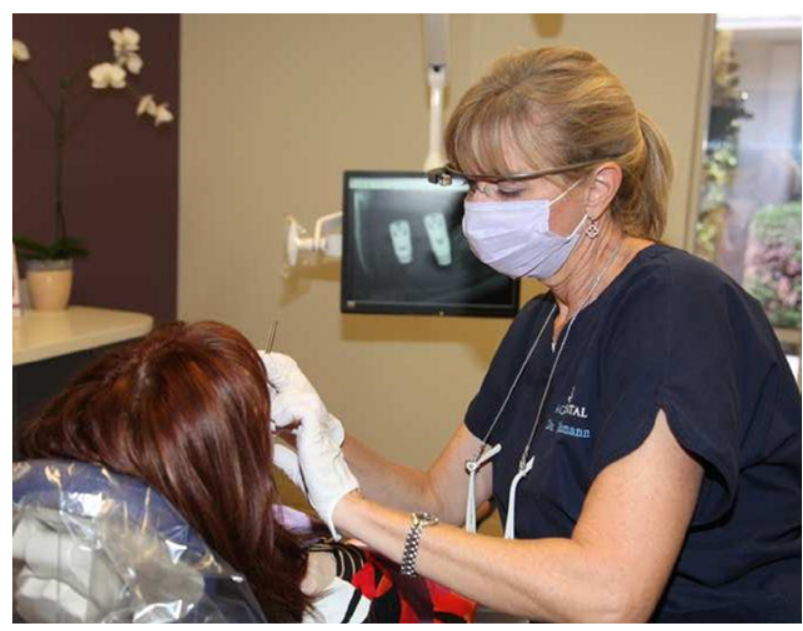

Figure 1: Using voice commands, Dr. Hamann uses smart glasses during the dental exam for teleconsultation with a physician concerning potential metal allergy in a patient with implant failure.

and antibiotics were prescribed with minimal resolution of symptoms. At week 3, exudate was expressed from the peri-implant tissues. The implants were removed, and granulomatous and necrotic tissues were removed by curettage and suction. The clinicians suspected that failure may be due to avascular necrosis, infection, or bio-incompatibility/ metal contact allergy to the implant. Suspecting that the patient may have experienced a hypersensitivity reaction to one of the metals present in the implants, the Agave dentists triaged the need for formal consult and allergy workup using real-time decision support through smart glasses. Though rare contact allergy to metals in oral implants has been reported $[12,13]$. Complete informed consent was obtained and smart glasses were used for hands-free, point-of-view, live video conferencing with a local patch testing physician. During this video-conference, the Agave dentist performed a brief oral exam and discussed the history and risk factors with the physician. Together it was agreed to patch test the patient to a metal screening patch test series prior to re-implantation.

\section{Case 2}

In another case, a 51-year-old Caucasian female with a history of mitral valve prolapse, jewelry avoidance, and prior positive patch test to gold and cobalt was examined for gingival irritation around 10 porcelain-fused-to-metal crowns. The crowns had been seated over the past 23 years and she had experienced red swollen gums around these crowns for at least 20 years. She also gave a history of a hypertrophic scar at the site of prior gold patch testing. The metal alloys of the crowns were predominantly high noble alloys containing gold, silver, platinum, palladium indium, tin, and gallium. On exam, the metal collars of the crowns had 1-2 mm circumferential gingival contact. Significant gingival inflammation was present surrounding each crown. Notably, the patient had excellent oral hygiene and there was no sub-gingival calculus and no signs of periodontal disease. Periodontal pocket depths were within normal limits. Given her past cobalt and gold patch test positivity and the frequent use of cobalt and gold in dental alloys, Agave dentists decided to consult a metal contact allergy specialist. Again, complete informed consent was obtained. The dentist donned the smart glasses and commenced a hands-free, point-of-view, live video conference with a contact allergy specialist. Together, it was agreed that one of the crowns should be replaced with a Lithium disilicate crown and the surrounding tissue be observed for resolution prior to more definitive treatment. The removed crown will be tested for cobaltrelease with a cobalt spot test and for gold and cobalt content with X-ray fluorescence spectrometry.

\section{Discussion}

Smart glasses (Google Glass) are a newly developed mobile technology composed of a miniature computer fitted into a pair of eyeglasses. Hands-free, voice controlled telecommunications technologies such as this are on the horizon for telemedicine and teledentistry. Our device, running the Google Android operating system, is equipped with a 5 megapixel 720p HD camera capable of highdefinition photo capturing and, simultaneously, video recording and streaming. The clinician is able to wear the device as it is fitted into comfortable eyewear containing a small translucent optical display. The hands-free and voice-activated device is well suited to use in dentistry and other procedural healthcare applications because if can be used during procedures that require gloves and instruments and it is compatible with rigorous infection-control standards. There are numerous advantages to a hands-free, point-of-view, video streaming, high-definition photo-taking and voice-activated telecommunication device. A clinician could use a digital camera or a cell phone, but the frequent use of both in a dental setting during an exam raise infection control concerns. As an example, a staff member could hold a smartphone with "video chat" connected to a consulting physician for the dentist during a dental exam, but demonstrating the lesions of concern would certainly interfere with the dentist's clinical exam and line-of-sight. The authors find the use of these smart glasses provide little interference and enable a useful live, two-way, photo- and video-augmented, hands-free, voice-activated method for enabling a multidisciplinary evaluation of complex patients that is not currently available to clinicians.

Coordination of care for patients with suspected oral metal allergy remains difficult and protracted. Many such patients have are sent to an oral surgeon, and then referred for patch testing. Though rarely patch testing may be performed on a first visit to a patch testing physician, generally patients are first seen by a the physician in an initial visit and then return to be patch tested when a staff member can have advance notice to prepare patch tests with extra series suited to the patient. Given these generalities, each of these patients' workups was likely saved at least two separate appointments.

In summary, this new technology has many potential applications in tele-health and allergy consultation, notably in the realm of oral metal allergy. In our case series, we have demonstrated its usefulness in coordinating care for patients with complex problems and the possibility to decrease the patients' burden for multiple visits.

Further studies are needed to assess its efficacy in other applications (Figure 1).

\section{References}

1. Tan E, Yung A, Jameson M, Oakley A, Rademaker M (2010) Successful triage of patients referred to a skin lesion clinic using teledermoscopy (IMAGE IT trial). Br J Dermatol 162: 803-811.

2. van der Heijden JP, de Keizer NF, Bos JD, Spuls PI, Witkamp L (2011) Teledermatology applied following patient selection by general practitioners in daily practice improves efficiency and quality of care at lower cost. $\mathrm{Br} J$ Dermatol 165: 1058-1065.

3. Khan SA, Omar H (2013) Teledentistry in practice: literature review. Telemed J E Health 19: 565-567.

4. Torres-Pereira CC, Morosini Ide A, Possebon RS, Giovanini AF, Bortoluzz 
Citation: Hamann CR, Hamann D, Sager K, Dameff C, Tully J, et al. (2014) Allergy Consultation via Smart Glasses: Cases Reports of Real-Time Clinical Decision Support for Suspected Oral Metal Allergy. J Allergy Ther 5: 186. doi:10.4172/2155-6121.1000186

Page 3 of 3

MC, et al. (2013) Teledentistry: distant diagnosis of oral disease using e-mails. Telemed J E Health 19: 117-121.

5. Ignatius E, Mäkelä K, Happonen R-P, Perälä S (2006) Teledentistry in dental specialist education in Finland. J Telemed Telecare 12: 46-49

6. Torres-Pereira C, Possebon RS, Simões A, Bortoluzzi MC, Leão JC, et al. (2008) Email for distance diagnosis of oral diseases: a preliminary study of teledentistry. J Telemed Telecare 14: 435-438.

7. Pappas $Y$, Atherton H, Sawmynaden P, Car J (2012) Email for clinical communication between healthcare professionals. Cochrane Database Syst Rev 9.

8. Park W, Lee HN, Jeong JS, Kwon JH, Lee GH, et al. (2012) Optimal protocol for teleconsultation with a cellular phone for dentoalveolar trauma: an in-vitro study. Imaging Sci Dent 42: 71-75.
9. Farber N, Haik J, Liran A, Weissman O, Winkler E (2011) Third generation cellular multimedia teleconsultations in plastic surgery. J Telemed Telecare 17: 199-202.

10. Chhabra N, Chhabra A, Jain RL, Kaur H, Bansal S (2011) Role Of Teledentistry In Dental Education: Need Of The Era. J Clin Diagnos Res 5: 1486-1488

11. Nickenig HJ, Wichmann M, Schlegel A, Eitner S (2008) Use of telemedicine for pre-implant dental assessment - a comparative study. J Telemed Telecare 14: 93-97.

12. Siddiqi A, Payne AG, De Silva RK, Duncan WJ (2011) Titanium allergy: could it affect dental implant integration? Clin Oral Implants Res 22: 673-680.

13. Sicilia A, Cuesta S, Coma G, Arregui I, Guisasola C, et al. (2008) Titanium allergy in dental implant patients: a clinical study on 1500 consecutive patients. Clin Oral Implants Res 19: 823-835
This article was originally published in a special issue, Skin Allergy handled by Editors. Dr. Anton B Alexandroff, University Hospitals of Leicester, UK, Dr.

Sharon E Jacob, University of California, USA. 